\title{
Stabilisation in Distribution by Delay Feedback Control for Hybrid Stochastic Differential Equations
}

\author{
Surong You, Liangjian Hu, Jianqiu Lu, Xuerong Mao
}

\begin{abstract}
This paper is concerned with the design of a feedback control based on past states in order to make a given unstable hybrid stochastic differential equation (SDE) to be stable in distribution (stabilisation in distribution). This is the first paper in this direction. Under the global Lipschitz condition on the coefficients of the given unstable hybrid SDE, we will show that the stabilisation in distribution can be achieved by linear delay feedback controls. In particular, we discuss how to design the feedback controls in two structure cases: state feedback and output injection.
\end{abstract}

Index Terms-Brownian motion, Markov chain, stability in distribution, delay feedback control.

AMS subject classifications-60H10, 60J10, $93 \mathrm{D} 15$.

\section{INTRODUCTION}

Hybrid systems have been widely used to model many practical systems in science and industry where the systems may experience abrupt changes in their structure and parameters (see, e.g., [2], [12], [25]). One important class of hybrid systems is the hybrid stochastic differential equations (SDEs), also known as SDEs with Markovian switching (see, e.g., [3], [4], [20], [21], [22], [28], [31]). An area of particular interest in the study of hybrid SDEs has been the analysis of stability. Most of the papers in this area are concerned with the stability of the trivial solution (equilibrium state) in the sense of $p$ th moment, probability 1 and so on (see, e.g., [5], [7], [8], [10], [15], [19], [23], [24], [30], [32]).

However, it is inappropriate to study the stability of the trivial solution but more appropriate to discuss the stability in distribution in many SDE models in the real world. For example, there is no equilibrium state to many SDE models in engineering including fault tolerant control systems, multiple target tracking, flexible manufacturing systems (see, e.g., [2], [4], [12], [22], [25]) and hence there is no point to discuss the stability of the trivial solution. The well-known mean

This work is entirely theoretical and the results can be reproduced using the methods described in this paper.

S. You is with Department of Statistics, Donghua University, Shanghai 201620, China. sryouedhu . edu. cn

L. Hu is with Department of Statistics, Donghua University, Shanghai 201620, China. 1 jhu@dhu. edu. cn

J. Lu is with Department of Statistics, Donghua University, Shanghai 201620, China. jianqiuluddhu. edu. cn

$\mathrm{X}$. Mao is with the Department of Mathematics and Statistics, University of Strathclyde, Glasgow G1 1XH, UK. Corresponding author. x.maodstrath.ac.uk reverting Ornstein-Uhlenbeck (OU) process in financial engineering (see, e.g., [8]) is described by a scalar SDE $d X(t)=\lambda(\mu-X(t)) d t+\sigma d B(t)$, where $\lambda, \mu, \sigma$ are all positive numbers and $B(t)$ is a scalar Brownian motion. This SDE does not have a trivial solution. However, the probability distribution of the solution $X(t)$ will converge to the normal distribution $N\left(\mu, \sigma^{2} / 2 \lambda\right)$ independent of the initial value $x(0) \in \mathbb{R}$ (see, e.g., [16, p.306]). For more information on the stability in distribution, we refer the reader to [4], [9], [21], [26], [27].

Consider a hybrid SDE

$$
d X(t)=f(X(t), r(t)) d t+g(X(t), r(t)) d B(t),
$$

where the state $X(t)$ takes values in $\mathbb{R}^{n}$ and the mode $r(t)$ is described by a Markov chain taking values in a finite space $\mathbb{S}=\{1,2, \cdots, N\}, B(t)$ is a Brownian motion, $f$ and $g$ are referred to as the drift and diffusion coefficient, respectively. If the given SDE does not have a desired property (e.g., stability), it is traditional (see, e.g., [20], [21], [28]) to design a feedback control $u(X(t), r(t))$, based on the current state $X(t)$ and mode $r(t)$, to make the controlled system

$$
\begin{aligned}
d X(t) & =[f(X(t), r(t))+u(X(t), r(t))] d t \\
& +g(X(t), r(t)) d B(t)
\end{aligned}
$$

to have the desired property. In this paper, we assume that the mode $r(t)$ is obvious at any time (and this is the case, for example, if the SDE (1.1) is a financial model where $r(t)$ stands for the interest rate [8]) but the state $x(t)$ is required to be observed. Due to an unavoidable time lag $\tau$ between the time when the observation of the state $x(t)$ is made and the time when the feedback control reaches the system, the control should be $u(X(t-\tau), r(t))$. Hence the controlled system should be in the form of a stochastic differential delay equation (SDDE)

$$
\begin{aligned}
d X(t) & =[f(X(t), r(t))+u(X(t-\tau), r(t))] d t \\
& +g(X(t), r(t)) d B(t) .
\end{aligned}
$$

In other words, (1.2) is theoretical while (1.3) is real. It is therefore absolutely necessary and important to study (1.3) for the real-world applications. Of course, when the mode $r(t)$ is not obvious and required to be observed, the control should become $u(X(t-\tau), r(t-\tau))$. The corresponding problem is more complicated and will be investigated in the future. 
If the desired property is the asymptotic stability of the trivial solution (equilibrium state) in the sense of either $p$ th moment or probability 1 , the stabilisation problem (1.3) has been studied by several authors (see, e.g., [6], [11], [18]). However, if the desired property is the asymptotic stability in distribution, the stabilisation problem (1.3) has not been studied yet. The latter is much harder than the former because the latter is concerned with the convergence of the probability distributions in the functional space $C\left([-\tau, 0] ; \mathbb{R}^{n}\right)$ (see Section 2 for the definition) while the former is to study if $\mathbb{E}|X(t)|^{p} \rightarrow 0$ or $X(t) \rightarrow 0$ almost surely. The mathematics developed for the latter in this paper is not only highly technical (please see, e.g., the proof of Theorem 3.4) but also very much different from the existing papers in this direction (e.g., [6], [11], [18]).

Before we develop our new theory on the stabilisation in distribution, let us highlight the key points we have made in this section:

- It is necessary and important to study the stabilisation in distribution for hybrid SDEs by delay feedback controls as there are real-world applications.

- The problem has not been studied yet is because the mathematics involved is highly technical.

\section{NOTATION}

Throughout this paper, unless otherwise specified, we let $\mathbb{R}^{n}$ be the $n$-dimensional Euclidean space and $\mathcal{B}\left(\mathbb{R}^{n}\right)$ denote the family of all Borel measurable sets in $\mathbb{R}^{n}$. If $x \in \mathbb{R}^{n}$, then $|x|$ is its Euclidean norm. Let $\tau$ be a positive number and $\mathcal{C}_{\tau}$ (or $C\left([-\tau, 0] ; \mathbb{R}^{n}\right)$ ) denote the family of continuous functions $\xi:[-\tau, 0] \rightarrow \mathbb{R}^{n}$ with norm $\|\xi\|_{\tau}=\sup _{-\tau \leq u \leq 0}|\xi(u)|$. Also, $\mathcal{B}\left(\mathcal{C}_{\tau}\right)$ denotes the family of all Borel measurable sets in $\mathcal{C}_{\tau}$. If $A$ is a vector or matrix, its transpose is denoted by $A^{T}$. If $A$ is a matrix, we let $|A|=\sqrt{\operatorname{trace}\left(A^{T} A\right)}$ be its trace norm and $\|A\|=\max \{|A x|:|x|=1\}$ be the operator norm. If $A$ is a symmetric matrix $\left(A=A^{T}\right)$, denote by $\lambda_{\min }(A)$ and $\lambda_{\max }(A)$ its smallest and largest eigenvalues, respectively. By $A>0$ and $A \geq 0$, we mean $A$ is positive and non-negative definite, respectively. If both $a, b$ are real numbers, then $a \wedge b=\min \{a, b\}$ and $a \vee b=\max \{a, b\}$.

We let $\left(\Omega, \mathcal{F},\left\{\mathcal{F}_{t}\right\}_{t \geq 0}, \mathbb{P}\right)$ be a complete probability space with a filtration $\left\{\overline{\mathcal{F}}_{t}\right\}_{t \geq 0}$ satisfying the usual conditions. For a subset $\bar{\Omega}$ of $\bar{\Omega}, I_{\bar{\Omega}}$ denotes its indicator function. Let $B(t)=\left(B_{1}(t), \cdots, B_{m}(t)\right)^{T}$ be an $m$ dimensional Brownian motion defined on the probability space. Let $r(t), t \geq 0$, be a right-continuous irreducible Markov chain on the probability space taking values in a finite state space $\mathbb{S}=\{1,2, \cdots, N\}$ with generator $\Gamma=\left(\gamma_{i j}\right)_{N \times N}$ given by

$\mathbb{P}\{r(t+\Delta)=j \mid r(t)=i\}= \begin{cases}\gamma_{i j} \Delta+o(\Delta) & \text { if } i \neq j, \\ 1+\gamma_{i i} \Delta+o(\Delta) & \text { if } i=j,\end{cases}$

where $\Delta>0$. Here $\gamma_{i j} \geq 0$ is the transition rate from $i$ to $j$ if $i \neq j$ while $\gamma_{i i}=-\sum_{j \neq i} \gamma_{i j}$. We assume that the Markov chain $r(\cdot)$ is independent of the Brownian motion $B(\cdot)$.

Consider an $n$-dimensional hybrid $\operatorname{SDE}$ (1.1) on $t \geq$ 0 , where $f: \mathbb{R}^{n} \times \mathbb{S} \rightarrow \mathbb{R}^{n}$ and $g: \mathbb{R}^{n} \times \mathbb{S} \rightarrow \mathbb{R}^{n \times m}$ are Borel measurable functions satisfying the following assumption.

Assumption 2.1: There is a pair of positive constants $a_{1}$ and $a_{2}$ such that

$$
\begin{aligned}
& |f(x, i)-f(y, i)|^{2} \leq a_{1}|x-y|^{2}, \\
& |g(x, i)-g(y, i)|^{2} \leq a_{2}|x-y|^{2}
\end{aligned}
$$

for all $x, y \in \mathbb{R}^{n}$ and $i \in \mathbb{S}$.

It is easy to see from Assumption 2.1 that

$$
|f(x, i)|^{2} \leq 2 a_{1}|x|^{2}+a_{0}, \quad|g(x, i)|^{2} \leq 2 a_{2}|x|^{2}+a_{0}
$$

for all $(x, i) \in \mathbb{R}^{n} \times \mathbb{S}$, where $a_{0}=2 \max _{i \in \mathbb{S}}\left(|f(0, i)|^{2} \vee\right.$ $\left.|g(0, i)|^{2}\right)$.

It is well known (see, e.g., [21]) that the hybrid SDE (1.1) has a unique global solution $X(t)$ on $t \geq 0$ for any given initial values $X(0) \in \mathbb{R}^{n}$ and $r(0) \in \mathbb{S}$. Assume that this given SDE does not have the desired property of stability in distribution and we are required to design a feedback control $u(X(t-\tau), r(t))$, to stabilise the system. To make the design simpler, we will seek a linear form of feedback control, namely $u(X(t-\tau), r(t))=$ $A(r(t)) X(t-\tau)$, where $A(i) \in \mathbb{R}^{n \times n}$ for all $i \in \mathbb{S}$, and we will often write $A(i)=A_{i}$. The underlying controlled system (1.3) therefore becomes

$$
\begin{aligned}
d X(t) & =(f(X(t), r(t))+A(r(t)) X(t-\tau)) d t \\
& +g(X(t), r(t)) d B(t) .
\end{aligned}
$$

Accordingly, our aim is to design $N$ matrices $A_{i}$ so that this controlled system is asymptotically stable in distribution.

The controlled system (2.2) is in fact a hybrid SDDE and it is therefore natural to impose the initial data

$$
\left\{\begin{array}{l}
\{X(u):-\tau \leq u \leq 0\}=\xi \in \mathcal{C}_{\tau}, \\
r(0)=i \in \mathbb{S} .
\end{array}\right.
$$

It is known (see, e.g., [13], [14], [21]) that under Assumption 2.1, the controlled SDDE (2.2) with the initial data (2.3) has a unique global solution on $t \geq-\tau$. Moreover, define $X_{t}=\{X(t+u):-\tau \leq u \leq 0\}$ for $t \geq 0$, which is a $\mathcal{C}_{\tau}$-valued process. When we need to emphasise the role of the initial data (2.3), we will write the solution by $X^{\xi, i}(t)$ while the Markov chain starting from $i$ at time 0 by $r^{i}(t)$. It is known (see, e.g., [21, Theorem 7.14 on p. 282]) that

$$
\mathbb{E}\left\|X_{t}^{\xi, i}\right\|^{2} \leq \kappa_{t}\left(1+\|\xi\|^{2}\right) \quad \forall t \geq 0
$$

where $\kappa_{t}$ is a positive constant dependent on $t$ but independent of the initial data $(\xi, i)$. It is also known that the joint process $\left(X_{t}, r(t)\right)$ on $t \geq 0$ forms a timehomogeneous Markov process on the state space $\mathcal{C}_{\tau} \times \mathbb{S}$. 
Define its transition probability measure on $\mathcal{C}_{\tau} \times \mathbb{S}$ by $p(t, \xi, i ; d \zeta \times\{j\})$. That is,

$$
\begin{aligned}
& \mathbb{P}\left(\left(X_{t}^{\xi, i}, r^{i}(t)\right) \in Z \times J\right) \\
= & \sum_{j \in J} \int_{Z} p(t, \xi, i ; d \zeta \times\{j\})
\end{aligned}
$$

for any $Z \in \mathcal{B}\left(\mathcal{C}_{\tau}\right)$ and $J \subset \mathbb{S}$.

Denote by $\mathcal{P}\left(\mathcal{C}_{\tau}\right)$ the family of probability measures on the measurable space $\left(\mathcal{C}_{\tau}, \mathcal{B}\left(\mathcal{C}_{\tau}\right)\right)$. For $P_{1}, P_{2} \in$ $\mathcal{P}\left(\mathcal{C}_{\tau}\right)$, define metric $d_{\mathbb{L}}$ by

$$
\begin{aligned}
& d_{\mathbb{L}}\left(P_{1}, P_{2}\right) \\
= & \sup _{\phi \in \mathbb{L}}\left|\int_{\mathcal{C}_{\tau}} \phi(\xi) P_{1}(d \xi)-\int_{\mathcal{C}_{\tau}} \phi(\xi) P_{2}(d \xi)\right|,
\end{aligned}
$$

where

$$
\begin{aligned}
\mathbb{L}= & \left\{\phi: \mathcal{C}_{\tau} \rightarrow \mathbb{R} \text { satisfying }|\phi(\xi)-\phi(\zeta)| \leq\|\xi-\zeta\|\right. \\
& \text { and } \left.|\phi(\xi)| \leq 1 \text { for } \xi, \zeta \in \mathcal{C}_{\tau}\right\} .
\end{aligned}
$$

Moreover, denote by $\mathcal{L}\left(X_{t}\right)$ the probability measure on $\left(\mathcal{C}_{\tau}, \mathcal{B}\left(\mathcal{C}_{\tau}\right)\right)$ generated by $X_{t}$.

Definition 2.2: The controlled system (2.2) is said to be asymptotically stable in distribution if there exists a probability measure $\mu_{\tau} \in \mathcal{P}\left(\mathcal{C}_{\tau}\right)$ such that

$$
\lim _{t \rightarrow \infty} d_{\mathbb{L}}\left(\mathcal{L}\left(X_{t}^{\xi, i}\right), \mu_{\tau}\right)=0
$$

for all $(\xi, i) \in \mathcal{C}_{\tau} \times \mathbb{S}$.

It should be pointed out that in the literature (see, e.g., [29]), the asymptotic stability in distribution is in general defined on the joint process $\left(X_{t}^{\xi, i}, r^{i}(t)\right)$, namely the transition probability measure $p(t, \xi, i ; d \zeta \times\{j\})$ will converge in distribution to a probability measure on $\mathcal{C}_{\tau} \times$ $\mathbb{S}$. On the other hand, given that the law of the Markov chain $r^{i}(t)$ is already known to converge to its unique stationary distribution (see, e.g., [1]), our definition here only on $X_{t}^{\xi, i}$ is consistent with that in the literature.

\section{MAin Results}

Assumption 3.1: There exists a positive number $b_{0}$ and $N$ symmetric positive definite matrices $W_{i}(1 \leq i \leq$ $N)$ such that

$$
\begin{aligned}
& \Psi(x, y, i) \\
& :=2(x-y)^{T} W_{i}\left[f(x, i)-f(y, i)+A_{i}(x-y)\right] \\
& +\operatorname{trace}\left[(g(x, i)-g(y, i))^{T} W_{i}(g(x, i)-g(y, i))\right] \\
& +\sum_{j=1}^{N} \gamma_{i j}(x-y)^{T} W_{j}(x-y) \leq-b_{0}|x-y|^{2}
\end{aligned}
$$

for all $(x, y, i) \in \mathbb{R}^{n} \times \mathbb{R}^{n} \times \mathbb{S}$.

We will explain in Section 4 how to design these desired matrices $A_{i}$ so that we can further identify $W_{i}$ 's and $b_{0}$ for this assumption to hold, but in this section we just assume this assumption is satisfied. It is straightforward to show from Assumptions 2.1 and 3.1 that

$$
\begin{aligned}
& \Phi(x, i) \\
& :=2 x^{T} W_{i}\left[f(x, i)+A_{i} x\right]+\operatorname{trace}\left[g(x, i)^{T} W_{i} g(x, i)\right] \\
& +\sum_{j=1}^{N} \gamma_{i j} x^{T} W_{j} x \leq-b_{0}|x|^{2}+b_{1}|x|+b_{2}
\end{aligned}
$$

for all $(x, i) \in \mathbb{R}^{n} \times \mathbb{S}$, where $b_{1}$ and $b_{2}$ are positive numbers. Throughout this paper, we will set

$$
a_{3}=\max _{i \in \mathbb{S}}\left\|A_{i}\right\|^{2} \text { and } a_{4}=\max _{i \in \mathbb{S}}\left\|W_{i} A_{i}\right\| .
$$

\section{A. Lyapunov functionals}

The key technique used in this paper is the method of Lyapunov functionals. To define the Lyapunov functionals, we introduce the segment $\hat{X}_{t}:=\{X(t+u)$ : $-2 \tau \leq u \leq 0\}$ for $t \geq \tau$. Please note that $\hat{X}_{t}$ is $C\left([-2 \tau, 0] ; \mathbb{R}^{n}\right)$-valued which is different from $X_{t}$. The Lyapunov functionals used in this paper will be of the form

$$
\begin{aligned}
& V\left(\hat{X}_{t}, r(t), t\right):=X^{T}(t) W_{r(t)} X(t) \\
& +\int_{t-\tau}^{t} \int_{s}^{t}\left[\theta_{1}|X(v)|^{2}+\theta_{2}|X(v-\tau)|^{2}\right] d v d s
\end{aligned}
$$

for $t \geq \tau$. Here $W_{i}$ 's are the matrices specified in Assumption 3.1 while $\theta_{1}$ and $\theta_{2}$ are two free positive numbers.

It is useful to observe that

$$
\begin{aligned}
& c_{1}|X(t)|^{2} \leq V\left(\hat{X}_{t}, r(t), t\right) \\
\leq & c_{2}|X(t)|^{2}+c_{3} \int_{t-2 \tau}^{t}|X(v)|^{2} d v
\end{aligned}
$$

where $c_{3}=\tau\left(\theta_{1} \vee \theta_{2}\right), c_{1}=\min _{i \in \mathbb{S}} \lambda_{\min }\left(W_{i}\right)$ and $c_{2}=$ $\max _{i \in \mathbb{S}} \lambda_{\max }\left(W_{i}\right)$.

Applying the generalised Itô formula (see, e.g., [21, Theorem 1.45 on p.48]) to the Lyapunov functional defined by (3.4) yields

$$
d V\left(\hat{X}_{t}, r(t), t\right)=L V\left(\hat{X}_{t}, r(t), t\right) d t+d M(t)
$$

for $t \geq \tau$, where

$$
\begin{aligned}
& L V\left(\hat{X}_{t}, r(t), t\right) \\
& =\Phi(X(t), r(t))-2 x^{T}(t) W_{r(t)} A_{r(t)}(X(t)-X(t-\tau)) \\
& +\theta_{1} \tau|X(t)|^{2}-\theta_{1} \int_{t-\tau}^{t}|X(s)|^{2} d s \\
& +\theta_{2} \tau|X(t-\tau)|^{2}-\theta_{2} \int_{t-\tau}^{t}|X(s-\tau)|^{2} d s
\end{aligned}
$$

and $M(t)$ is a martingale with $M(0)=0$ (whose form is of no use in this paper). Making use of (3.2) and introducing the third free positive number $\theta_{3} \in\left(0, b_{0} / a_{4}\right)$, 
we get from (3.7) that

$$
\begin{aligned}
& L V\left(\hat{X}_{t}, r(t), t\right) \\
& \leq-\left(b_{0}-a_{4} \theta_{3}-\theta_{1} \tau\right)|X(t)|^{2}+b_{1}|X(t)|+b_{2} \\
& +\left(a_{4} / \theta_{3}\right)|X(t)-X(t-\tau)|^{2}-\theta_{1} \int_{t-\tau}^{t}|X(s)|^{2} d s \\
& +\theta_{2} \tau|X(t-\tau)|^{2}-\theta_{2} \int_{t-2 \tau}^{t-\tau}|X(s-\tau)|^{2} d s
\end{aligned}
$$

for $t \geq \tau$. On the other hand, we can derive from (2.2) along with (2.1) and (3.3) that

$$
\begin{aligned}
& \mathbb{E}|X(t)-X(t-\tau)|^{2} \\
\leq & 2 a_{0} \tau(2 \tau+1)+4\left(2 \tau a_{1}+a_{2}\right) \int_{t-\tau}^{t} \mathbb{E}|X(s)|^{2} d s \\
+ & 4 \tau a_{3} \int_{t-2 \tau}^{t-\tau} \mathbb{E}|X(s)|^{2} d s .
\end{aligned}
$$

We therefore obtain that

$$
\begin{aligned}
& \mathbb{E}\left(L V\left(\hat{X}_{t}, r(t), t\right)\right) \\
& \leq-\left(b_{0}-a_{4} \theta_{3}-\theta_{1} \tau\right) \mathbb{E}|X(t)|^{2}+b_{1} \mathbb{E}|X(t)| \\
& +b_{2}+2 a_{0} a_{4} \tau(2 \tau+1) / \theta_{3}+\theta_{2} \tau \mathbb{E}|X(t-\tau)|^{2} \\
& -\left[\theta_{1}-4 a_{4}\left(2 \tau a_{1}+a_{2}\right) / \theta_{3}\right] \int_{t-\tau}^{t} \mathbb{E}|X(s)|^{2} d s \\
& -\left(\theta_{2}-4 \tau a_{3} a_{4} / \theta_{3}\right) \int_{t-2 \tau}^{t-\tau} \mathbb{E}|X(s)|^{2} d s
\end{aligned}
$$

for $t \geq \tau$. Throughout of this paper, we define the set of three free parameters

$$
\begin{gathered}
\Theta=\left\{\left(\theta_{1}, \theta_{2}, \theta_{3}\right): \theta_{1} \theta_{3}>4 a_{2} a_{4}, \theta_{2}>0,\right. \\
\left.\theta_{3} \in\left(0, b_{0} / a_{4}\right)\right\}
\end{gathered}
$$

and let

$$
\tau^{*}=\sup _{\left(\theta_{1}, \theta_{2}, \theta_{3}\right) \in \Theta} \frac{b_{0}-a_{4} \theta_{3}}{\theta_{1}+\theta_{2}} \wedge \frac{\theta_{1} \theta_{3}-4 a_{2} a_{4}}{8 a_{1} a_{4}} \wedge \frac{\theta_{2} \theta_{3}}{4 a_{3} a_{4}} .
$$

These are technical parameters. In particular, the meaning of $\tau^{*}$ will become clear later (see Theorem 3.4).

\section{B. Lemmas}

Lemma 3.2: Let Assumptions 2.1 and 3.1 hold. If $\tau<\tau^{*}$, then the solution of equation (2.2) with the initial data (2.3) satisfies

$$
\mathbb{E}\left\|X_{t}^{\xi, i}\right\|^{2} \leq \bar{\theta}\left(1+\|\xi\|^{2}\right)
$$

for all $t \geq 0$, where $\bar{\theta}$ is a positive number independent of initial data $(\xi, i)$.

Proof. As $\tau<\tau^{*}$, we can choose three positive parameters $\left(\theta_{1}, \theta_{2}, \theta_{3}\right) \in \Theta$ for

$$
\tau<\frac{b_{0}-a_{4} \theta_{3}}{\theta_{1}+\theta_{2}} \wedge \frac{\theta_{1} \theta_{3}-4 a_{2} a_{4}}{8 a_{1} a_{4}} \wedge \frac{\theta_{2} \theta_{3}}{4 a_{3} a_{4}} .
$$

Fix the initial data $(\xi, i)$ arbitrarily and write $X^{\xi, i}(t)=$ $X(t)$ for simplicity. It then follows from (3.10) that

$$
\begin{aligned}
& \mathbb{E}\left(L V\left(\hat{X}_{t}, r(t), t\right)\right) \\
& \leq \bar{\theta}_{0}+b_{1} \mathbb{E}|X(t)|-\bar{\theta}_{1} \mathbb{E}|X(t)|^{2}+ \\
& +\bar{\theta}_{2} \mathbb{E}|X(t-\tau)|^{2}-\bar{\theta}_{3} \int_{t-2 \tau}^{t} \mathbb{E}|X(s)|^{2} d s
\end{aligned}
$$

for $t \geq \tau$, where $\bar{\theta}_{0}=b_{2}+2 a_{0} a_{4} \tau(2 \tau+1) / \theta_{3}, \bar{\theta}_{1}=$ $b_{0}-a_{4} \theta_{3}-\theta_{1} \tau, \bar{\theta}_{2}=\theta_{2} \tau$ and $\bar{\theta}_{3}=\left[\theta_{1}-4 a_{4}\left(2 \tau a_{1}+\right.\right.$ $\left.\left.a_{2}\right) / \theta_{3}\right] \wedge\left(\theta_{2}-4 \tau a_{3} a_{4} / \theta_{3}\right)$. We see from (3.14) that they are all positive and $\bar{\theta}_{1}>\bar{\theta}_{2}$. Let $\bar{\theta}_{4}=\left(\bar{\theta}_{1}-\bar{\theta}_{2}\right) / 2$. Noting that

$$
\bar{\theta}_{0}+b_{1} \mathbb{E}|X(t)|-\bar{\theta}_{4} \mathbb{E}|X(t)|^{2} \leq \bar{\theta}_{5}:=\bar{\theta}_{0}+\frac{b_{1}^{2}}{4 \bar{\theta}_{4}^{2}},
$$

we get from (3.15) that

$$
\begin{aligned}
& \mathbb{E}\left(L V\left(\hat{X}_{t}, r(t), t\right)\right) \\
& \leq \bar{\theta}_{5}-\left(\bar{\theta}_{2}+\bar{\theta}_{4}\right) \mathbb{E}|X(t)|^{2}+ \\
& +\bar{\theta}_{2} \mathbb{E}|X(t-\tau)|^{2}-\bar{\theta}_{3} \int_{t-2 \tau}^{t} \mathbb{E}|X(s)|^{2} d s
\end{aligned}
$$

for $t \geq \tau$. Let $\bar{\theta}_{6}>0$ be sufficiently small for

$$
c_{3} \bar{\theta}_{6} \leq \bar{\theta}_{3} \text { and } \bar{\theta}_{2}+\bar{\theta}_{4} \geq \bar{\theta}_{2} e^{\bar{\theta}_{6} \tau}+c_{2} \bar{\theta}_{6},
$$

where $c_{1}-c_{3}$ have been defined below (3.5). The existence of $\bar{\theta}_{6}$ is clear as $\bar{\theta}_{2}, \bar{\theta}_{3}, \bar{\theta}_{4}$ etc. are all positive. Applying the generalised Itô formula (see, e.g., [21, Theorem 1.14 on page 48]) to $e^{\bar{\theta}_{6} t} V\left(\hat{X}_{t}, r(t), t\right)$, we have

$$
\begin{aligned}
& e^{\bar{\theta}_{6} t} \mathbb{E}\left(V\left(\hat{X}_{t}, r(t), t\right)\right)-e^{\bar{\theta}_{6} \tau} \mathbb{E}\left(V\left(\hat{X}_{\tau}, r(\tau), \tau\right)\right) \\
& =\int_{\tau}^{t} e^{\bar{\theta}_{6} s} \mathbb{E}\left(\bar{\theta}_{6} V\left(\hat{X}_{s}, r(s), s\right)+L V\left(\hat{X}_{s}, r(s), s\right)\right) d s
\end{aligned}
$$

for $t \geq \tau$. Making use of (2.4) and (3.5), we can then easily obtain

$$
\begin{aligned}
& c_{1} e^{\bar{\theta}_{6} t} \mathbb{E}|X(t)|^{2}-\bar{\theta}_{7}\left(1+\|\xi\|^{2}\right) \\
& \leq \int_{\tau}^{t} e^{\bar{\theta}_{6} s} \mathbb{E}\left(c_{2} \bar{\theta}_{6}|X(s)|^{2}+c_{3} \bar{\theta}_{6} \int_{s-2 \tau}^{s}|X(u)|^{2} d u\right. \\
& \left.\quad+L V\left(\hat{X}_{s}, r(s), s\right)\right) d s
\end{aligned}
$$

where $\bar{\theta}_{7}$ and following $\bar{\theta}_{8}$ etc. are all positive numbers independent of $(\xi, i)$. Noting that

$$
\begin{aligned}
& \int_{\tau}^{t} e^{\bar{\theta}_{6} s} \mathbb{E}|X(s-\tau)|^{2} d s \\
\leq & \bar{\theta}_{8}\left(1+\|\xi\|^{2}\right)+e^{\bar{\theta}_{6} \tau} \int_{\tau}^{t} e^{\bar{\theta}_{6} s} \mathbb{E}|X(s)|^{2} d s,
\end{aligned}
$$

we can then obtain from (3.18) that

$$
\begin{aligned}
& c_{1} e^{\bar{\theta}_{6} t} \mathbb{E}|X(t)|^{2}-\bar{\theta}_{7}\left(1+\|\xi\|^{2}\right) \\
& \leq \int_{\tau}^{t} e^{\bar{\theta}_{6} s} \bar{\theta}_{9}\left(1+\|\xi\|^{2}\right) d s \leq e^{\bar{\theta}_{6} t}\left(\bar{\theta}_{9} / \bar{\theta}_{6}\right)\left(1+\|\xi\|^{2}\right) .
\end{aligned}
$$


This implies that $\mathbb{E}|X(t)|^{2} \leq \bar{\theta}_{10}\left(1+\|\xi\|^{2}\right)$ for al $t \geq \tau$. But, it is easy to show that for $t \geq 2 \tau$,

$$
\begin{aligned}
& \mathbb{E}\left\|X_{t}\right\|^{2} \leq 3|X(t)|^{2}+6 a_{0} \tau^{2} \\
& +\left[6 \tau\left(a_{1}+a_{3}\right)+12 a_{2}\right] \int_{t-\tau}^{t} \mathbb{E}|X(s)|^{2} d s \\
& \leq \bar{\theta}_{11}\left(1+\|\xi\|^{2}\right),
\end{aligned}
$$

where $\bar{\theta}_{11}=2 \bar{\theta}_{10}+6 a_{0} \tau^{2}+2 \tau \bar{\theta}_{10}\left[6 \tau\left(a_{1}+a_{3}\right)+12 a_{2}\right]$. This, together with (2.4), shows that the required assertion (3.13) must hold. The proof is hence complete.

Lemma 3.3: Let Assumptions 2.1 and 3.1 hold. If $\tau<\tau^{*}$, then for any $(\xi, \zeta, i) \in \mathcal{C}_{\tau} \times \mathcal{C}_{\tau} \times \mathbb{S}$,

$$
\mathbb{E}\left\|X_{t}^{\xi, i}-X_{t}^{\zeta, i}\right\|^{2} \leq \beta_{1}\|\xi-\zeta\|^{2} e^{-\beta_{2} t}
$$

for all $t \geq 2 \tau$, where both $\beta_{1}$ and $\beta_{2}$ are positive numbers independent of $(\xi, \zeta, i)$.

Proof. As $\tau<\tau^{*}$, we can choose three positive numbers $\left(\theta_{1}, \theta_{2}, \theta_{3}\right) \in \Theta$ such that

$$
\theta_{1} \theta_{3}>2 a_{2} a_{4}, \theta_{3} \in\left(0, b_{0} / a_{4}\right)
$$

and

$$
\tau<\frac{b_{0}-a_{4} \theta_{3}}{\theta_{1}+\theta_{2}} \wedge \frac{\theta_{1} \theta_{3}-2 a_{2} a_{4}}{4 a_{1} a_{4}} \wedge \frac{\theta_{2} \theta_{3}}{4 a_{3} a_{4}} .
$$

Fix any $(\xi, \zeta, i) \in \mathcal{C}_{\tau} \times \mathcal{C}_{\tau} \times \mathbb{S}$ and set $Z(t)=X^{\xi, i}(t)-$ $X^{\zeta, i}(t)$ for $t \geq-\tau$. So $Z_{t}=\{Z(t+u):-\tau \leq u \leq 0\}$ for $t \geq 0$ while $\hat{Z}_{t}=\{Z(t+u):-2 \tau \leq u \leq 0\}$ for $t \geq \tau$. We will use the Lyapunov functional defined by (3.4) by replacing $\hat{X}_{t}$ with $\hat{Z}_{t}$, namely $V\left(\hat{Z}_{t}, r(t), t\right)$. By the generalised Itô formula, we can show that

$$
d V\left(\hat{Z}_{t}, r(t), t\right)=L V\left(\hat{Z}_{t}, r(t), t\right) d t+d \bar{M}(t)
$$

for $t \geq \tau$, where

$$
\begin{aligned}
& L V\left(\hat{Z}_{t}, r(t), t\right)=\Psi\left(X^{\xi, i}(t), X^{\zeta, i}, r(t)\right) \\
& -2 Z^{T}(t) W_{r(t)} A_{r(t)}(Z(t)-Z(t-\tau)) \\
& +\theta_{1} \tau|Z(t)|^{2}-\theta_{1} \int_{t-\tau}^{t}|Z(s)|^{2} d s \\
& +\theta_{2} \tau|Z(t-\tau)|^{2}-\theta_{2} \int_{t-\tau}^{t}|Z(s-\tau)|^{2} d s
\end{aligned}
$$

and $\bar{M}(t)$ is a martingale with $\bar{M}(0)=0$ (see, e.g., [21, Theorem 1.14 on page 48]). Making use of Assumption 3.1 , we then get

$$
\begin{aligned}
& L V\left(\hat{Z}_{t}, r(t), t\right) \\
& \leq-\left(b_{0}-a_{4} \theta_{3}-\theta_{1} \tau\right)|Z(t)|^{2} \\
& +\left(a_{4} / \theta_{3}\right)|Z(t)-Z(t-\tau)|^{2}-\theta_{1} \int_{t-\tau}^{t}|Z(s)|^{2} d s \\
& +\theta_{2} \tau|Z(t-\tau)|^{2}-\theta_{2} \int_{t-2 \tau}^{t-\tau}|Z(s)|^{2} d s
\end{aligned}
$$

for $t \geq \tau$. But, in the same way as (3.9) was proved, we can show that

$$
\begin{gathered}
\mathbb{E}|Z(t)-Z(t-\tau)|^{2} \leq 2\left(2 \tau a_{1}+a_{2}\right) \int_{t-\tau}^{t} \mathbb{E}|Z(s)|^{2} d s \\
+4 \tau a_{3} \int_{t-2 \tau}^{t-\tau} \mathbb{E}|Z(s)|^{2} d s
\end{gathered}
$$

We hence have that

$$
\begin{aligned}
\mathbb{E}\left(L V\left(\hat{Z}_{t}, r(t), t\right)\right) & \leq-\beta_{3} \mathbb{E}|Z(t)|^{2}+\beta_{4} \mathbb{E}|Z(t-\tau)|^{2} \\
& -\beta_{5} \int_{t-2 \tau}^{t} \mathbb{E}|Z(s)|^{2} d s
\end{aligned}
$$

for $t \geq \tau$, where $\beta_{3}=b_{0}-a_{4} \theta_{3}-\theta_{1} \tau, \beta_{4}=\theta_{2} \tau$, $\beta_{5}=\left[\theta_{1}-2 a_{4}\left(2 \tau a_{1}+a_{2}\right) / \theta_{3}\right] \wedge\left[\theta_{2}-4 \tau a_{3} a_{4} / \theta_{3}\right]$. By conditions (3.20) and (3.21), we see $\beta_{3}>\beta_{4}>0$ and $\beta_{5}>0$. Starting from here, we can show, in the same way as Lemma 3.2 was proved, that

$$
\mathbb{E}|Z(t)|^{2} \leq \beta_{6}\|\xi-\zeta\|^{2} e^{-\beta_{2} t}
$$

for all $t \geq \tau$, where $\beta_{2}$ and $\beta_{6}$ are all positive numbers independent of $(\xi, \zeta, i)$. However, it is straightforward to show that for $t \geq 2 \tau$,

$$
\mathbb{E}\left\|Z_{t}\right\|^{2} \leq 4\left[\tau\left(a_{1}+\alpha_{3}\right)+a_{2}\right] \int_{t-\tau}^{t} \mathbb{E}|Z(s)|^{2} d s .
$$

Substituting (3.27) into (3.28) yields

$$
\mathbb{E}\left\|Z_{t}\right\|^{2} \leq \beta_{1}\|\xi-\zeta\|^{2} e^{-\beta_{2} t}, \quad \forall t \geq 2 \tau,
$$

where $\beta_{1}=4 \beta_{6}\left[\tau\left(a_{1}+\alpha_{3}\right)+a_{2}\right] e^{\beta_{2} \tau}$. This is the required assertion (3.19). The proof is therefore complete.

\section{Key theorem}

Theorem 3.4: Let Assumptions 2.1 and 3.1 hold. If $\tau<\tau^{*}$, then there exists a unique probability measure $\mu_{\tau} \in \mathcal{P}\left(\mathcal{C}_{\tau}\right)$ such that

$$
\lim _{t \rightarrow \infty} d_{\mathbb{L}}\left(\mathcal{L}\left(X_{t}^{\xi, i}\right), \mu_{\tau}\right)=0
$$

for all $(\xi, i) \in \mathcal{C}_{\tau} \times \mathbb{S}$. In other words, the controlled system (2.2) is asymptotically stable in distribution provided $\tau<\tau^{*}$.

Proof. The proof is very technical so is divided into three steps in order to make it more understandable. $\mathcal{K}$ of $\mathcal{C}_{\tau}$

Step 1. We first claim that for any compact subset

$$
\lim _{t \rightarrow \infty} d_{\mathbb{L}}\left(\mathcal{L}\left(X_{t}^{\xi, i}\right), \mathcal{L}\left(X_{t}^{\zeta, j}\right)\right)=0
$$

uniformly in $(\xi, \zeta, i, j) \in \mathcal{K} \times \mathcal{K} \times \mathbb{S} \times \mathbb{S}$. Define the stopping time $\kappa_{i j}=\inf \left\{t: r^{i}(t)=r^{j}(t), t \geq 0\right\}$. Then $\kappa_{i j}<\infty$ a.s. by the ergodic property of the Markov chain (see, e.g., [1]). Hence, for any $\varepsilon \in(0,1)$, there is a positive number $T_{1}>0$ such that

$$
\mathbb{P}\left(\kappa_{i j} \leq T_{1}\right)>1-\varepsilon / 6 \quad \forall i, j \in \mathbb{S} .
$$

Recalling a known result (see, e.g., [21, Theorem 7.14 on p. 282]) that

$$
\sup _{(\xi, i) \in \mathcal{K} \times \mathbb{S}} \mathbb{E}\left(\sup _{-\tau \leq t \leq T_{1}}\left|X^{\xi, i}(t)\right|^{2}\right)<\infty,
$$

we see there is a sufficiently large $h>0$ such that

$$
\mathbb{P}\left(\Omega_{\xi, i}\right)>1-\varepsilon / 12 \quad \forall(\xi, i) \in \mathcal{K} \times \mathbb{S},
$$


where $\Omega_{\xi, i}=\left\{\omega \in \Omega: \sup _{-\tau \leq t \leq T_{1}}\left|X^{\xi, i}(t, \omega)\right| \leq h\right\}$. We now fix $\xi, \zeta \in \mathcal{K}$ and $i, j \in \mathbb{S}$ arbitrarily. For any $\phi \in \mathbb{L}$ and $t \geq T_{1}$, we have

$$
\left|\mathbb{E} \phi\left(X_{t}^{\xi, i}\right)-\mathbb{E} \phi\left(X_{t}^{\zeta, j}\right)\right| \leq \frac{\varepsilon}{3}+H_{1}(t),
$$

where

$$
H_{1}(t):=\mathbb{E}\left(I_{\left\{\kappa_{i j} \leq T_{1}\right\}}\left|\phi\left(X_{t}^{\xi, i}\right)-\phi\left(X_{t}^{\zeta, j}\right)\right|\right) .
$$

Set $\Omega_{1}=\Omega_{\xi, i} \cap \Omega_{\zeta, j} \cap\left\{\kappa_{i j} \leq T_{1}\right\}$. By the time homogeneous property of equation (2.2), we derive

$$
\begin{aligned}
& H_{1}(t) \\
= & \mathbb{E}\left(I_{\left\{\kappa_{i j} \leq T_{1}\right\}} \mathbb{E}\left(\left|\phi\left(X^{\xi, i}(t)\right)-\phi\left(X^{\zeta, j}(t)\right)\right| \mid \mathcal{F}_{\kappa_{i j}}\right)\right) \\
= & \mathbb{E}\left(I_{\left\{\kappa_{i j} \leq T_{1}\right\}} \mathbb{E} \mid \phi\left(X^{\bar{\xi}, l}\left(t-\kappa_{i j}\right)\right)-\phi\left(X^{\bar{\zeta}, l}\left(t-\kappa_{i j}\right) \mid\right)\right. \\
\leq & \frac{\varepsilon}{3}+\mathbb{E}\left(I_{\Omega_{1}} \mathbb{E}\left|X^{\bar{\xi}, l}\left(t-\kappa_{i j}\right)-X^{\bar{\zeta}, l}\left(t-\kappa_{i j}\right)\right|\right),(3.35)
\end{aligned}
$$

where $\bar{\xi}=X_{\kappa_{i j}}^{\xi, i}, \bar{\zeta}=X_{\kappa_{i j}}^{\zeta, j}$ and $l=r^{i}\left(\kappa_{i j}\right)=r^{j}\left(\kappa_{i j}\right)$. Observing that $\|\bar{\xi}\| \vee\|\bar{\zeta}\| \leq h$ for any $\omega \in \Omega_{1}$, we can apply Lemma 3.3 to see that there is another positive constant $T_{2}$ such that

$$
\mathbb{E}\left|X^{\bar{\xi}, l}\left(t-\kappa_{i j}\right)-X^{\bar{\zeta}, l}\left(t-\kappa_{i j}\right)\right| \leq \frac{\varepsilon}{3}, \quad \forall t \geq T_{1}+T_{2}
$$

whenever $\omega \in \Omega_{1}$. Using this and (3.35), we obtain from (3.34) that

$$
\left|\mathbb{E} \phi\left(X_{t}^{\xi, i}\right)-\mathbb{E} \phi\left(X_{t}^{\zeta, j}\right)\right| \leq \varepsilon, \quad \forall t \geq T_{1}+T_{2} .
$$

Since $\phi$ is arbitrary, we must have

$$
d_{\mathbb{L}}\left(\mathcal{L}\left(X_{t}^{\xi, i}\right), \mathcal{L}\left(X_{t}^{\zeta, j}\right)\right) \leq \varepsilon, \quad \forall t \geq T_{1}+T_{2}
$$

for all $(\xi, \zeta, i, j) \in \mathcal{C}_{\tau} \times \mathcal{C}_{\tau} \times \mathbb{S} \times \mathbb{S}$. This proves (3.31).

Step 2. We next claim that for any $(\xi, i) \in \mathcal{C}_{\tau} \times \mathbb{S}$, $\left\{\mathcal{L}\left(X_{t}^{\xi, i}\right)\right\}_{t \geq 0}$ is a Cauchy sequence in $\mathcal{P}\left(\mathcal{C}_{\tau}\right)$ with metric $d_{\mathbb{L}}$. In other words, we need to show that for any $\varepsilon>0$, there is a positive number $T_{3}$ such that

$$
d_{\mathbb{L}}\left(\mathcal{L}\left(X_{v+u}^{\xi, i}\right), \mathcal{L}\left(X_{u}^{\xi, i}\right)\right) \leq \varepsilon
$$

for all $u \geq T_{3}$ and $v>0$. Let $\varepsilon \in(0,1)$ be arbitrarily. By Lemma 3.2, there is a $\bar{h}>0$ such that

$$
\mathbb{P}\left\{\omega \in \Omega:\left\|X_{v}^{\xi, i}(\omega)\right\| \leq \bar{h}\right\}>1-\varepsilon / 4 \quad \forall v>0 .
$$

For any $\phi \in \mathbb{L}$ and $u, v>0$, we can then derive, using (2.5) and (3.38), that

$$
\begin{aligned}
& \left|\mathbb{E} \phi\left(X_{v+u}^{\xi, i}\right)-\mathbb{E} \phi\left(X_{u}^{\xi, i}\right)\right| \\
= & \left|\mathbb{E}\left(\mathbb{E}\left[\phi\left(X_{v+u}^{\xi, i}\right) \mid \mathcal{F}_{v}\right]\right)-\mathbb{E} \phi\left(X_{u}^{\xi, i}\right)\right| \\
= & \left|\sum_{j \in \mathbb{S}} \int_{\mathcal{C}_{\tau}} \mathbb{E} \phi\left(X_{u}^{\zeta, j}\right) p(v, \xi, i ; d \zeta \times\{j\})-\mathbb{E} \phi\left(X_{u}^{\xi, i}\right)\right| \\
\leq & \sum_{j \in \mathbb{S}} \int_{\mathcal{C}_{\tau}}\left|\mathbb{E} \phi\left(X_{u}^{\zeta, j}\right)-\mathbb{E} \phi\left(X_{u}^{\xi, i}\right)\right| p(v, \xi, i ; d \zeta \times\{j\}) \\
\leq & \left.\frac{\varepsilon}{2}+\sum_{j \in \mathbb{S}} \int_{Z_{\bar{h}}} d_{\mathbb{L}}\left(\mathcal{L}\left(X_{u}^{\zeta, j}\right)\right), \mathcal{L}\left(X_{u}^{\xi, i}\right)\right) p(v, \xi, i ; d \zeta \times\{j\}),
\end{aligned}
$$

where $Z_{\bar{h}}=\left\{\zeta \in \mathcal{C}_{\tau}:\|\zeta\| \leq \bar{h}\right\}$. But, by (3.31), there is a positive integer $T_{3}$ such that

$$
\left.d_{\mathbb{L}}\left(\mathcal{L}\left(X_{u}^{\zeta, j}\right)\right), \mathcal{L}\left(X_{u}^{\xi, i}\right)\right) \leq \frac{\varepsilon}{2}, \quad \forall u \geq T_{3}
$$

whenever $(\zeta, j) \in Z_{\bar{h}} \times \mathbb{S}$. We therefore obtain

$$
\left|\mathbb{E} \phi\left(X_{v+u}^{\xi, i}\right)-\mathbb{E} \phi\left(X_{u}^{\xi, i}\right)\right| \leq \varepsilon
$$

for $u \geq T_{3}$ and $v>0$. As this holds for any $\phi \in \mathbb{L}$, we must have (3.37) as claimed.

Step 3. It follows from Step 2 that there is a unique $\mu_{\tau} \in \mathcal{P}\left(\mathcal{C}_{\tau}\right)$ such that

$$
\lim _{t \rightarrow \infty} d_{\mathbb{L}}\left(\mathcal{L}\left(X_{t}^{0,1}\right), \mu_{\tau}\right)=0 .
$$

This, together with (3.31), implies that

$$
\begin{aligned}
\lim _{t \rightarrow \infty} d_{\mathbb{L}}\left(\mathcal{L}\left(X_{t}^{\xi, i}\right), \mu_{\tau}\right) & \leq \lim _{t \rightarrow \infty} d_{\mathbb{L}}\left(\mathcal{L}\left(X_{t}^{\xi, i}\right), \mathcal{L}\left(X_{t}^{0,1}\right)\right) \\
& +\lim _{t \rightarrow \infty} d_{\mathbb{L}}\left(\mathcal{L}\left(X_{t}^{0,1}\right), \mu_{\tau}\right)=0
\end{aligned}
$$

for all $(\xi, i) \in \mathcal{C}_{\tau} \times \mathbb{S}$, which is the desired assertion (3.30). The proof is therefore complete.

\section{Design of matrices $A_{i}$}

The use of our main result, Theorem 3.4, depends on the design of matrices $A_{i}(i \in \mathbb{S})$. In this section we will explain how to design these matrices in the situation of structure feedback controls. That is, we will look for the matrices in the structure form of $A_{i}=F_{i} G_{i}$ with $F_{i} \in \mathbb{R}^{n \times l}$ and $G_{i} \in \mathbb{R}^{l \times n}$ for some positive integer $l$. We will discuss two cases which are known as: (i) state feedback; (ii) output injection (see, e.g., [18]).

(i) State feedback: design $F_{i}$ 's when $G_{i}$ 's are given

We will use the technique of linear matrix inequalities (LMIs, see, e.g., [30]) to design $F_{i}$ 's in this subsection. Under Assumption 2.1, we will design $F_{i}$ 's in two steps.

Step 4.1: Find $N$ pairs of symmetric matrices $W_{i}$ and $\hat{W}_{i}(i \in \mathbb{S})$ with $W_{i}>0$ such that

$$
\begin{aligned}
& 2(x-y)^{T} W_{i}[f(x, i)-f(y, i)] \\
+ & \operatorname{trace}\left[(g(x, i)-g(y, i))^{T} W_{i}(g(x, i)-g(y, i))\right] \\
\leq & (x-y)^{T} \hat{W}_{i}(x-y)
\end{aligned}
$$

for all $(x, y, i) \in \mathbb{R}^{n} \times \mathbb{R}^{n} \times \mathbb{S}$.

There are lots of choices for $W_{i}$ and $\hat{W}_{i}$. For example, it will do if we choose any symmetric positivedefinite matrices $W_{i}$ and then let $\hat{W}_{i}=\left(2 \sqrt{a_{1}}+\right.$ $\left.a_{2}\right)\left\|W_{i}\right\| I_{n}$ with $I_{n}$ being $n \times n$ identity matrix. However, it is wise to choose matrices in order to make use of the given structures of $f$ and $g$ so that the second step can be made more easily.

Step 4.2: Find a solution of matrices $F_{i}$ to the LMIs

$$
\hat{W}_{i}+F_{i} G_{i}+G_{i}^{T} F_{i}^{T}+\sum_{j=1}^{N} \gamma_{i j} W_{j}<0, \quad i \in \mathbb{S} .
$$


Please note that one can use Matlab to search for the solution matrices. The following corollary is obvious.

Corollary 4.3: Under Assumption 2.1, find matrices $F_{i}(i \in \mathbb{S})$ as described in Steps 4.1 and 4.2. Then Assumption 3.1 is satisfied with $A_{i}=F_{i} G_{i}$ and

$$
b_{0}=-\max _{i \in \mathbb{S}} \lambda_{\max }\left(\hat{W}_{i}+F_{i} G_{i}+G_{i}^{T} F_{i}^{T}+\sum_{j=1}^{N} \gamma_{i j} W_{j}\right) \text {. }
$$
given

(ii) Output injection: design $G_{i}$ 's when $F_{i}$ 's are

This is very similar to the case of state feedback. We describe it as another corollary.

Corollary 4.4: Under Assumption 2.1, find matrices $W_{i}$ and $\hat{W}_{i}(i \in \mathbb{S})$ as Step 4.1 describes and then find a solution of matrices $G_{i}$ to the LMIs (4.2). Then Theorem 3.4 holds with $A_{i}=F_{i} G_{i}$ and $b_{0}$ being the same as in Corollary 4.3.

\section{EXAMPLE}

We will discuss an example in this section to illustrate our theory.

\section{Example 5.1: Consider a second order SDE}

$$
\ddot{z}(t)=-2 \dot{z}(t)+0.21 z(t)+\left[\alpha_{r(t)}+\sigma_{r(t)} z(t)\right] \dot{B}(t),
$$

where $\dot{B}(t)$ is a scalar white noise (informally thought as the derivative of a scalar Brownian motion $B(t)), r(t)$ is a Markov chain taking values in the state space $\mathbb{S}=\{1,2\}$ with the generator

$$
\Gamma=\left(\begin{array}{cc}
-1 & 1 \\
2 & -2
\end{array}\right)
$$

and the coefficients are specified by $\alpha_{1}=2, \alpha_{2}=$ $-1, \sigma_{1}=0.5, \sigma_{2}=1$. This $\operatorname{SDE}$ describes a hybrid stochastic oscillator (see, e.g., [16]). Introducing $X(t)=\left(X_{1}(t), X_{2}(t)\right)^{T}=(z(t), \dot{z}(t))^{T}$, we can write the oscillator as the two-dimensional linear hybrid SDE

$$
d X(t)=H X(t) d t+\left[k_{r(t)}+K_{r(t)} X(t)\right] d B(t),
$$

where

$$
H=\left(\begin{array}{cc}
0 & 1 \\
0.21 & -2
\end{array}\right), k_{i}=\left(\begin{array}{c}
0 \\
\alpha_{i}
\end{array}\right), K_{i}=\left(\begin{array}{cc}
0 & 0 \\
\sigma_{i} & 0
\end{array}\right) .
$$

Given any initial value $X(0) \in \mathbb{R}^{2}$, the mean of the solution to equation (5.1) has the form

$$
\mathbb{E} X(t)=e^{H t} X(0),
$$

where

$e^{H t}=\left(\begin{array}{cc}\frac{21}{22} e^{-2.1 t}+\frac{1}{22} e^{0.1 t} & -\frac{5}{11} e^{-2.1 t}+\frac{5}{11} e^{0.1 t} \\ -\frac{21}{220} e^{-2.1 t}+\frac{21}{220} e^{0.1 t} & \frac{21}{22} e^{-2.1 t}+\frac{1}{22} e^{0.1 t}\end{array}\right)$.

It then follows, for example, $\mathbb{E} X_{1}(t) \rightarrow \infty$ and $\mathbb{E} X_{2}(t) \rightarrow \infty$ when $X(0)=(1,0)^{T}$ while $\mathbb{E} X_{1}(t) \rightarrow$ $-\infty$ and $\mathbb{E} X_{2}(t) \rightarrow-\infty$ when $X(0)=(-1,0)^{T}$. These show that equation (5.2) is not stable in distribution.
Let us now apply our new theory to design a delay feedback control to stabilise the SDE. Due to the page limit, we only discuss a structure feedback control in the following interesting situation, where

- only $X_{1}$-component, in both modes, can be observed and the control can only be fed into $d X_{1}$-component.

In terms of mathematics, our control function has the form $A_{i} X(t-\tau)$ with

$$
A_{i}=\left(\begin{array}{cc}
-\beta_{i} & 0 \\
0 & 0
\end{array}\right),
$$

where $\beta_{1}$ and $\beta_{2}$ are both positive numbers to be chosen. Namely, the controlled system has the form

$$
\begin{aligned}
d X(t) & =\left[H X(t)+A_{r(t)} X(t-\tau) d t\right. \\
& +\left[k_{r(t)}+K_{r(t)} X(t)\right] d B(t) .
\end{aligned}
$$

It is straightforward to see that if we let $W_{i}$ be the identity matrix for both $i=1$ and 2, then Assumption 3.1 holds as long as

$$
\begin{aligned}
& -b_{0}=\lambda_{\max }\left(H+A_{i}+H^{T}+A_{i}^{T}+K_{i}^{T} K_{i}\right) \\
& =\lambda_{\max }\left(\left(\begin{array}{cc}
-2 \beta_{i}+\sigma_{i}^{2} & 1.21 \\
1.21 & -4
\end{array}\right)\right)<0 .
\end{aligned}
$$

Setting $-2 \beta_{i}+\sigma_{i}^{2}=-4$ for $i=1,2$, namely

$$
-2 \beta_{1}+0.25=-4, \quad-2 \beta_{2}+1=-4,
$$

we get $\beta_{1}=2.125$ and $\beta_{2}=2.5$. Consequently, Assumption 3.1 holds with $b_{0}=2.79$. It is also easy to check that Assumption (2.1) holds with $a_{1}=2.244$ and $a_{2}=1$. Moreover, by (3.3), we compute $a_{3}=6.25$ and $a_{4}=2.5$. Then (3.11) becomes

$$
\begin{gathered}
\Theta=\left\{\left(\theta_{1}, \theta_{2}, \theta_{3}\right): \theta_{1} \theta_{3}>10, \theta_{2}>0,\right. \\
\left.\theta_{3} \in(0,1.116)\right\}
\end{gathered}
$$

and, by (3.12),

$$
\tau^{*}=\sup _{\left(\theta_{1}, \theta_{2}, \theta_{3}\right) \in \Theta} \frac{2.79-2.5 \theta_{3}}{\theta_{1}+\theta_{2}} \wedge \frac{\theta_{1} \theta_{3}-10}{44.88} \wedge \frac{\theta_{2} \theta_{3}}{62.5} .
$$

Choosing $\theta_{3}=0.5$ and setting

$$
\tau_{1}^{*}=\frac{1.54}{\theta_{1}+\theta_{2}}=\frac{0.5 \theta_{1}-10}{44.88}=\frac{0.5 \theta_{2}}{62.5},
$$

we get $\theta_{1}=24.49471, \theta_{2}=6.259339$ and $\tau_{1}^{*}=$ 0.05006471 . As $\left(\theta_{1}, \theta_{2}, \theta_{3}\right) \in \Theta$, we must have $\tau_{1}^{*} \leq \tau^{*}$. By Theorem 3.4, we can then conclude that for each $\tau<$ 0.05006471 , there exists a unique probability measure $\mu_{\tau} \in \mathcal{P}\left(C\left([-\tau, 0] ; \mathbb{R}^{2}\right)\right)$ such that the solution of the controlled system (5.5) satisfies

$$
\lim _{t \rightarrow \infty} d_{\mathbb{L}}\left(\mathcal{L}\left(X_{t}^{\xi, i}\right), \mu_{\tau}\right)=0
$$

for all $(\xi, i) \in C\left([-\tau, 0] ; \mathbb{R}^{2}\right) \times \mathbb{S}$. 


\section{CONCLUSion}

In this paper we initiated the new problem of stabilisation in distribution by delay feedback controls for a class of nonlinear hybrid SDEs whose drift and diffusion coefficients are globally Lipschitz continuous. We successfully showed that the stabilisation in distribution can be achieved by linear delay feedback controls. In particular, we discuss how to design the feedback controls in two structure cases: state feedback and output injection. We also obtain a positive $\tau^{*}$ so that the delay feedback control works as long as $\tau \leq \tau^{*}$. Although $\tau^{*}$ obtained is not optimal yet, it can be determined numerically so that our theory can be applied more easily in practice. A hybrid stochastic oscillator (i.e., a 2-dimensional hybrid $\mathrm{SDE}$ ) was discussed in order to illustrate our theory.

\section{ACKNOWLEDGEMENTS}

The authors would like to thank the associate editor and reviewers for their very professional comments and suggestions. The authors would also like to thank the Royal Society (WM160014, Royal Society Wolfson Research Merit Award), the Royal Society and the Newton Fund (NA160317, Royal Society-Newton Advanced Fellowship), the EPSRC (EP/K503174/1), China Fundamental Research Funds for the Central Universities (2232020D-37) for their financial support.

\section{REFERENCES}

[1] Anderson, W.J., Continuous-Time Markov Chains, Springer, New York, 1991.

[2] Athans, M., Command and control (C2) theory: A challenge to control science, IEEE Trans. Automat. Contr. 32 (1987), 286-293.

[3] Bahar, A. and Mao, X., Stochastic delay population dynamics, Int. J. Pure Appl. Math. 11(4) (2004), 377-400.

[4] Basak, G.K., Bisi, A. and Ghosh, M.K., Stability of a random diffusion with linear drift, J. Math. Anal. Appl. 202 (1996), 604622.

[5] Fei, C., Fei, W., Mao, X., Xia, D. and Yan, L., Stabilisation of highly nonlinear hybrid systems by feedback control based on discrete-time state observations, IEEE Trans. Automat. Control. 65(7) (2020), 2899-2912.

[6] Hu, J., Liu, W., Deng, F. and Mao, X., Advances in stabilisation of hybrid stochastic differential equations by delay feedback control, SIAM J. Control Optim. 58(2) (2020), 735-754.

[7] Ji, Y. and Chizeck, H.J., Controllability, stabilizability and continuous-time Markovian jump linear quadratic control, IEEE Trans. Automat. Control 35 (1990), 777-788.

[8] Lewis, A.L., Option Valuation under Stochastic Volatility: with Mathematica Code, Finance Press, 2000.

[9] Li, X., Ma, Q., Yang, H. and Yuan, C. , The numerical invariant measure of stochastic differential equations with Markovian switching, SIAM Journal on Numerical Analysis 56 (3) (2019), $1435-1456$.

[10] Li, X. and Mao, X., Stabilisation of highly nonlinear hybrid stochastic differential delay equations by delay feedback control, Automatica 112 (2020), 108657, 11 pp.

[11] Lu, Z., Hu, J. and Mao, X., Stabilisation by delay feedback control for highly nonlinear hybrid stochastic differential equations, Discrete Contin. Dyn. Syst. Ser. B. 24(8) (2019), 4099-4116.

[12] Luo, Q. and Mao, X., Stochastic Population dynamics under regime switching II, J. Math. Anal. Appl. 355 (2009), 577-593.

[13] Mao, X., Stability of Stochastic Differential Equations with Respect to Semimartingales, Longman Scientific and Technical, 1991.

[14] Mao, X., Exponential Stability of Stochastic Differential Equations, Marcel Dekker, 1994.
[15] Mao, X., Stability of stochastic differential equations with Markovian switching, Stochastic Process. Appl. 79 (1999), 45-67.

[16] Mao, X., Stochastic Differential Equations and Their Applications, 2nd Edition, Elsevie, 2007.

[17] Mao, X., Stationary distribution of stochastic population systems, Systems Control Lett. 60 (2011), 398-406.

[18] Mao, X., Lam, J. and Huang, L., Stabilisation of hybrid stochastic differential equations by delay feedback control, Systems Control Lett. 57 (2008), 927-936.

[19] Mao, X., Matasov, A. and Piunovskiy, A.B., Stochastic differential delay equations with Markovian switching, Bernoulli 6(1) (2000), 73-90.

[20] Mao, X., Yin, G. and Yuan, C., Stabilization and destabilization of hybrid systems of stochastic differential equations, Automatica 43 (2007), 264-273.

[21] Mao, X. and Yuan, C., Stochastic Differential Equations with Markovian Switching, Imperial College Press, 2006.

[22] Mariton, M., Jump Linear Systems in Automatic Control, Marcel Dekker, 1990.

[23] Shaikhet, L., Stability of stochastic hereditary systems with Markov switching, Theory Stoch. Process. 2(18) (1996), 180-184.

[24] Sun, M., Lam, J., Xu, S. and Zou, Y., Robust exponential stabilization for Markovian jump systems with mode-dependent input delay, Automatica J. IFAC 43 (2007), 1799-1807.

[25] Sworder, D.D. and Robinson, V.G., Feedback regulators for jump parameter systems with state and control depend transition rates, IEEE Trans.Automat. Control 18 (1973), 355-360.

[26] Wu, F., Yin, G. and Mei, H., Stochastic functional differential equations with infinite delay: Existence and uniqueness of solutions, solution maps, markov properties, and ergodicity, Journal of Differential Equations 262 (2017), 1226- 1252.

[27] Yuan, C. and Mao, X., Asymptotic stability in distribution of stochastic differential equations with Markovian switching, Sto. Proce. Their Appl. 103 (2003), 277-291.

[28] Yuan, C. and Mao, X., Robust stability and controllability of stochastic differential delay equations with Markovian switching, Automatica J. IFAC 40(3) (2004), 343-354.

[29] Yuan, C., Zou, J. and Mao, X, Stability in distribution of stochastic differential delay equations with Markovian switching, Systems Control Lett. 50 (2003), 195-207.

[30] Yue, D. and Han, Q., Delay-dependent exponential stability of stochastic systems with time-varying delay, nonlinearity, and Markovian switching, IEEE Trans. Automat. Control 50 (2005), 217-222.

[31] Zhu, E., Wang, Y., Wang, Y. et al., Stability Analysis of Recurrent Neural Networks with Random Delay and Markovian Switching, J Inequal Appl 2010, 191546 (2010).

[32] Zhu, E., Tian, X. and Wang, Y., On pth moment exponential stability of stochastic differential equations with Markovian switching and time-varying delay, J Inequal Appl 2015, 137 (2015). 